\title{
Metagenomic data of three Covid19 patients submitted by NIAID Bioinformatics and Computational Biosciences Branch is from the gut - and not the lungs (BALF) as stated: Muribaculum is an intestinal microbe
}

Sandeep Chakraborty

\section{Letter}

The Covid19 anaerobic hypothesis states that SARS-Cov2 is enabling anaerobic bacteria to colonize the lungs, leading to Lemierre's syndrome like symptoms [1]. However, the metagenomic data from 3 Covid19 patients (age $\mathrm{M}=40, \mathrm{~F}=20, \mathrm{~F}=31$ ) submitted by NIAID Bioinformatics and Computational Biosciences Branch (Accid:PRJNA631287) is from the gut - and not lungs (BALF) as stated (Table 1). The Registration date of this sequencing data is : 8-May-2020. Muribaculum is an intestinal microbe [2] - and the microbe composition can be seen to be completely different from that in the lungs.

The data size is very small - 5000 reads per patient. So this should be very easy to verify.

\section{References}

1. Chakraborty S (2020). Sars-cov2 enables anaerobic bacteria (Prevotella, et al) to colonize the lungs disrupting homeostasis - symptoms (ARDS, septic shock, blood clots, arterial stroke) finds resonance,

Table 1: Metagenomic data from 3 Covid19 patients submitted by NIAID Bioinformatics and Computational Biosciences Branch is from the gut - and not lungs (BALF) as stated Muribaculum is an intestinal microbe [2] - and the microbe composition can be seen to be completely different from the lung microbiome. There are 5000 reads per patient.

\begin{tabular}{|c|c|c|}
\hline $\mathrm{M}=40$ yrs & $\mathrm{F}=20$ yrs & $\mathrm{F}=31$ yrs \\
\hline Muribaculum-2411 & Muribaculum-1460 & Muribaculum-1925 \\
Bacteroidales-539 & Clostridium-519 & Bacteroidales-576 \\
Clostridium-474 & Bacteroides-454 & Bacteroides-419 \\
Bacteroides-191 & Acetatifactor-364 & Clostridium-379 \\
Parabacteroides-136 & Kineothrix-327 & Anaerobacterium-222 \\
Kineothrix-130 & Bacteroidales-302 & Kineothrix-189 \\
Lactobacillus-122 & Oscillibacter-273 & Parabacteroides-177 \\
Butyricimonas-108 & Parabacteroides-218 & Lactobacillus-148 \\
Ruminococcus-89 & Butyricimonas-169 & Butyricimonas-131 \\
Anaerobacterium-81 & Alistipes-111 & Ruminococcus-74 \\
Anaerocolumna-75 & Eubacterium-102 & Peptococcus-70 \\
Acetatifactor-72 & Ruminococcus-85 & Vallitalea-60 \\
Eubacterium-47 & Flintibacter-76 & Anaerocolumna-58 \\
Oscillibacter-47 & Sporobacter-51 & Pseudobacteroides-55 \\
Breznakia-46 & Pseudoflavonifractor-50 & Eubacterium-44 \\
Alistipes-40 & Peptococcus-39 & Oscillibacter-39 \\
Flintibacter-37 & Phocea-38 & Acetatifactor-37 \\
\hline
\end{tabular}


with key differences, in the 'forgotten disease' Lemierre syndrome, caused by anaerobic bacteria enabled by Epstein Barr virus. doi:10.31219/osf.io/usztn. URL osf.io/usztn.

2. Lagkouvardos I, Lesker TR, Hitch TC, Gálvez EJ, Smit N, et al. (2019) Sequence and cultivation study of muribaculaceae reveals novel species, host preference, and functional potential of this yet undescribed family. Microbiome 7: 28. 


\begin{tabular}{|l|l|}
\hline env_local_scale & \multicolumn{2}{l}{ hompital ENVO:00002173 } \\
\hline env_medium & mucus material ENVO:02000040 \\
\hline geo_loc_name_country & USA \\
\hline geo_loc_name_country_continent & North America \\
\hline geo_loc_name & USA:MD,Bethesda \\
\hline host_disease & COVID-19 \\
\hline Host & Homo sapiens \\
\hline Instrument & Illumina MiSeq \\
\hline isolation_source & bronchoalveolar lavage fluid(BALF)
\end{tabular}

Figure 1: Annotation of these samples as BALF: This can be viewed here: https://www.ncbi.nlm.nih.gov/Traces/study/?acc=SRP260836 